\title{
Hospitalizations for Emergent Medical, Surgical, and Obstetric Conditions in Boston During the COVID-19 Pandemic
}

J Gen Intern Med 35(10):3129-32

DOI: $10.1007 / \mathrm{s} 11606-020-06027-2$

(c) Society of General Internal Medicine 2020

\section{INTRODUCTION}

The coronavirus disease 2019 (COVID-19) pandemic has resulted in a decline in patients seeking outpatient medical care. ${ }^{1,2}$ How COVID-19 has impacted patients seeking care for emergent conditions remains unclear, with emerging studies demonstrating a reduction in acute cardiovascular hospitalizations. ${ }^{3,4}$ In this study, we examined the incidence of emergent medical, surgical, and obstetric hospitalizations at a tertiary referral center in Boston, MA.

\section{METHODS}

We identified all hospital admissions from a 651-bed hospital in Boston between January 1, 2019, and April 25, 2020. Primary discharge diagnoses were collected from hospital administrative databases and categorized using Agency for Healthcare Research and Quality Clinical Classification Software. ${ }^{5}$ COVID-19 hospitalizations were identified based on positive polymerase chain reaction testing or COVID-19specific ICD-10 diagnoses coding (U071).

We examined the weekly incidence of overall admissions to medical, surgical, obstetric, and psychiatric services as well as hospitalizations for COVID-19 in 2020. We then examined the incidence of hospitalizations for emergent conditions including acute medical conditions, acute surgical conditions, chronic disease exacerbations, obstetric conditions, and COVID-19. We used $t$ tests with two-sided $P$ values $<.05$ for significance to compare the incidence of hospitalizations in the 6 weeks following the Boston COVID-19 public health emergency declaration (week of March 11, 2020) with the same period in 2019. We then conducted time series analyses comparing weekly hospitalization rates in the period prior to COVID-19 (January 1, 2019 to March 10, 2020) with the COVID-19 period (March 11, 2020 to April 28, 2020) using ordinary least squares regressions with Newey-West standard

Received June 10, 2020

Accepted June 30, 2020

Published online July 22, 2020 errors to account for autocorrelation. Analyses were conducted using Stata 14.1. This research was classified as exempt by the Beth Israel Deaconess Medical Center institutional review board.

\section{RESULTS}

Data from 12,935 hospitalizations from January 1, 2020, through April 28, 2020, were examined, including 7165 (55.4\%) medical, 3189 (24.7\%) surgical, 1807 (14.0\%) obstetric, 175 (1.4\%) psychiatric, and 599 (4.7\%) COVID-19 hospitalizations. In total, 4840 (37.4\%) hospitalizations were classified as emergent (Fig. 1).

Compared with the same 6-week period in 2019, there was a $35.1 \%$ decrease in weekly hospitalizations overall and $44.7 \%$ decrease in weekly non-COVID hospitalizations in the COVID-19 period. There were significantly fewer weekly hospitalizations for acute medical conditions $(-51.0 \%)$, acute surgical conditions $(-30.5 \%)$, chronic disease exacerbations $(-55.0 \%)$, and obstetric hospitalizations $(-12.9 \%)$ (Table 1$)$.

In time series analysis, there were no significant trends in weekly hospitalization rates in the pre-COVID-19 period. During the week of the Boston public health emergency declaration, there was an immediate decrease in hospitalizations, overall, for acute medical conditions, and for chronic disease exacerbations. During the COVID period, there was a continued increase in COVID-19 hospitalizations and a decline in hospitalizations for acute surgical conditions, for chronic disease exacerbations, and for obstetric conditions (Table 1).

\section{DISCUSSION}

In this single-center study at a tertiary care center in Boston, we observed an immediate decline in the incidence of hospitalizations overall and for a variety of emergent medical and surgical conditions which corresponded with the initiation of local public health emergency measures, and this decline persisted throughout the next 6 weeks. These findings build on recent studies demonstrating a reduction in the incidence of acute cardiovascular hospitalizations ${ }^{3,4}$ and a study which examined a smaller subset of acute conditions in the Veterans Affairs health system. ${ }^{6}$ 
a Overall hospitalizations

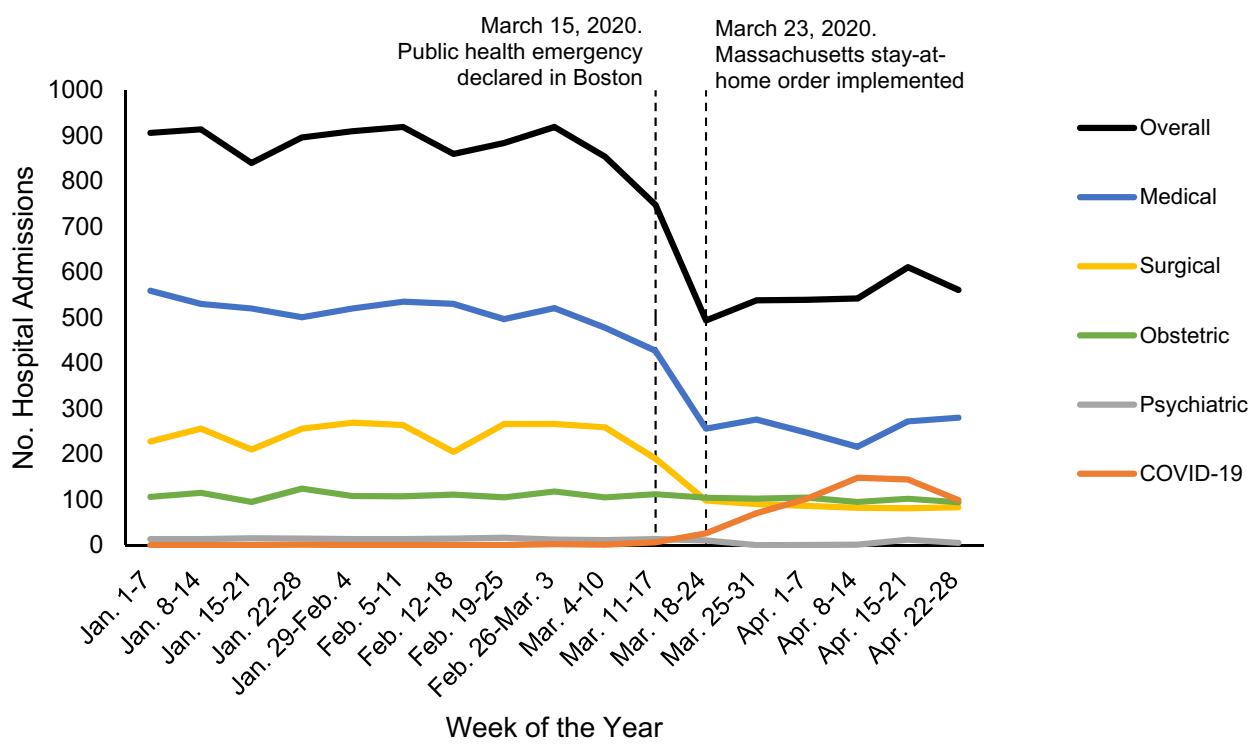

b Emergent hospitalizations

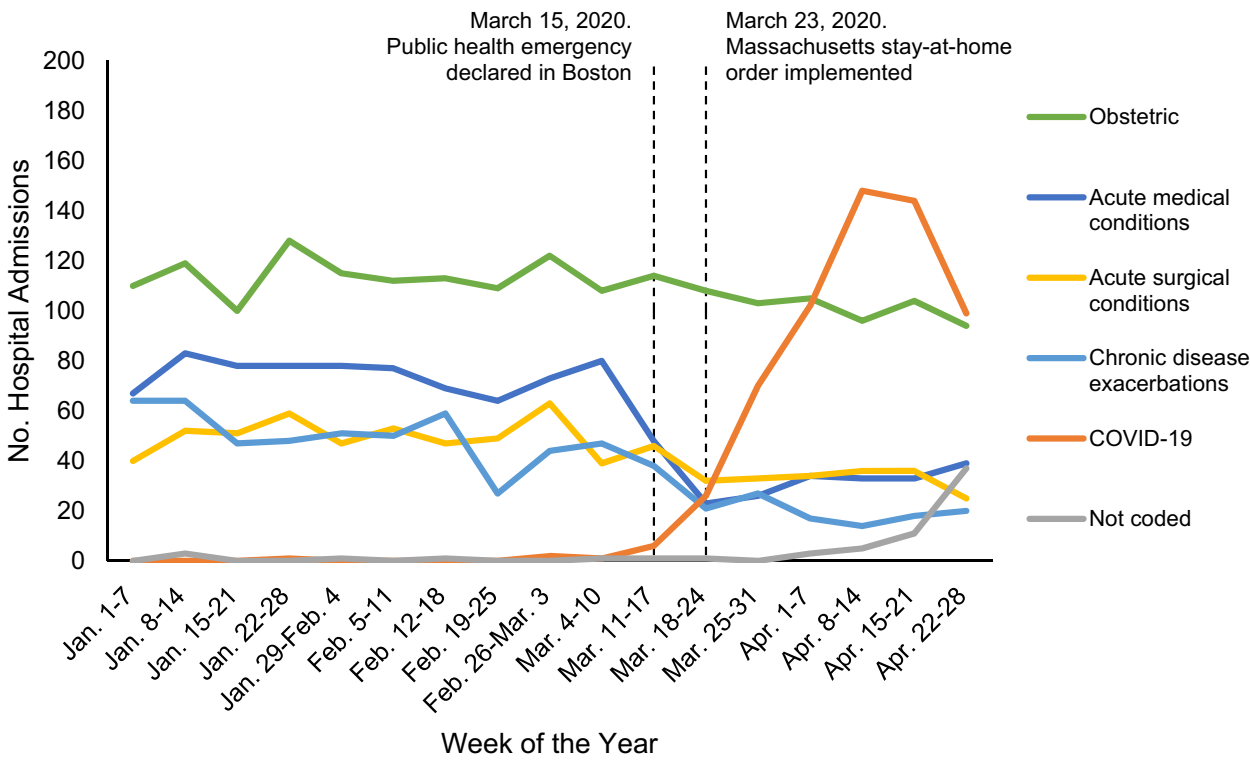

Figure 1 Weekly trends in hospitalizations. a Overall hospitalizations. b Emergent hospitalizations. Note: Overall hospitalizations categorized by specialty of discharging service. COVID-19 hospitalizations were identified by an International Statistical Classification of Diseases and Related Health Problems, $10^{\text {th }}$ revision (ICD-10) code of "U071 COVID-19, virus identified" or positive COVID-19 polymerase chain reaction testing during hospitalization or up to 14 days prior to hospitalization. Emergent conditions categorized using AHRQ Clinical Classification Software Refined categorization of ICD-10 primary discharge diagnosis codes. Acute medical conditions include acute myocardial infarction; cardiac arrest and ventricular fibrillation; cardiac dysrhythmias; arterial dissections; endocarditis and endocardial disease; myocarditis and cardiomyopathy; pericarditis and pericardial disease; acute hemorrhagic cerebrovascular disease; cerebral infarction; transient cerebral ischemia; gastrointestinal hemorrhage; acute pulmonary embolism; acute phlebitis, thrombophlebitis; and thromboembolism. Acute surgical conditions include appendicitis and other appendiceal conditions; gastrointestinal and biliary perforation; intestinal obstruction and ileus; and peritonitis and intra-abdominal abscess, and initial encounters for traumatic injuries including fractures, dislocations, traumatic brain and spinal cord injury, open wounds, crush injuries, and burns. Chronic disease exacerbations include hospitalizations for asthma, chronic obstructive pulmonary disease and bronchiectasis, diabetes, and heart failure. A total of 93 patients $(0.7 \%)$ were categorized as "Not coded" due to missing discharge diagnosis codes, as 37 remained hospitalized at the time of data collection and 56 lacked billing data.

This study relied on administrative coding and cannot explain the reasons for the observed reduction in the incidence of emergent hospitalizations which are likely multifactorial and may include individuals not seeking hospital care, individuals choosing to seek care at other hospitals, competing risk of hospitalization from COVID-19, geographic migration from Boston, and a reduction in underlying events. 
Table 1 Time Series Analysis

\begin{tabular}{|c|c|c|c|c|c|c|c|}
\hline & \multicolumn{4}{|c|}{$\begin{array}{l}\text { Historical comparison, mean no. admissions } \\
\text { per week }\end{array}$} & \multicolumn{3}{|c|}{ Time series analysis, no. admissions per week ( $95 \%$ CI) } \\
\hline & $\begin{array}{l}03 / 11 / 19 \\
\text { to } 04 / 28 / 19\end{array}$ & $\begin{array}{l}03 / 11 / 20 \\
\text { to } 04 / 28 / 20\end{array}$ & $\begin{array}{l}\% \\
\text { difference }\end{array}$ & $\begin{array}{l}P \\
\text { value }\end{array}$ & $\begin{array}{l}\text { Rate of change } \\
\text { pre-COVID } \\
\text { period: } 01 / 01 / 19 \\
\text { to } 03 / 10 / 20\end{array}$ & $\begin{array}{l}\text { Immediate change in } \\
\text { level: week of } 03 / 11 / 19\end{array}$ & $\begin{array}{l}\text { Rate of change COVID } \\
\text { period: } 03 / 11 / 19 \text { to } 04 \text { / } \\
28 / 20\end{array}$ \\
\hline Overall hospitalizations & $887.4(38.2)$ & $576.1(83.4)$ & $-35.1 \%$ & $<.001$ & $-0.1(-0.5$ to 0.3$)$ & $-292.6(-404.6$ to -180.7$)$ & $-11.5(-41.1$ to 18.0$)$ \\
\hline Excluding COVID-19 & $887.4(38.2)$ & $491.1(113.9)$ & $-44.7 \%$ & .001 & $-0.1(-0.5$ to 0.3$)$ & $-354.7(-509.6$ to -199.9$)$ & -32.7 ( -68.6 to 3.2$)$ \\
\hline COVID-19 hospitalizations & $0.0(0.0)$ & $85.0(54.6)$ & N/A & $<.001$ & $0.0(0.0$ to 0.0$)$ & $62.1(-4.5$ to 128.6$)$ & $21.2(9.3$ to 33.0$)$ \\
\hline Acute medical conditions & $68.9(5.8)$ & $33.7(8.2)$ & $-51.0 \%$ & $<.001$ & $0.1(0.0$ to 0.2$)$ & $-34.8(-46.4$ to -23.2$)$ & $0.0(-3.1$ to 3.1$)$ \\
\hline Cardiovascular events $\mathrm{s}^{\mathrm{a}}$ & $40.3(4.2)$ & $15.0(4.3)$ & $-62.8 \%$ & $<.001$ & $0.0(-0.1$ to 0.1$)$ & $-23.1(-28.5$ to -17.6$)$ & $-0.7(-2.1$ to 0.7$)$ \\
\hline Cerebrovascular events $^{\mathrm{b}}$ & $16.0(4.5)$ & $10.6(3.5)$ & $-33.9 \%$ & .03 & $0.1(0.0$ to 0.1$)$ & $-8.6(-14.6$ to -2.6$)$ & $1.1(0.4$ to 1.8$)$ \\
\hline Venous thromboembolism & $6.7(1.9)$ & $4.4(3.3)$ & $-34.0 \%$ & .14 & $0.0(0.0$ to 0.0$)$ & $-1.5(-4.1$ to 1.1$)$ & $0.5(-0.8$ to 1.7$)$ \\
\hline Gastrointestinal hemorrhage & $5.9(2.5)$ & $3.7(3.0)$ & $-36.6 \%$ & .18 & $0.0(0.0$ to 0.1$)$ & $-1.7(-4.7$ to 1.3$)$ & $-0.8(-1.7$ to 0.1$)$ \\
\hline Acute surgical conditions & $49.7(6.2)$ & $34.6(6.3)$ & $-30.5 \%$ & $<.001$ & $-0.1(-0.2$ to 0.0$)$ & $-6.9(-15.7$ to 1.9$)$ & $-1.9(-3.5$ to -0.3$)$ \\
\hline Intra-abdominal emergencies ${ }^{\mathrm{c}}$ & $12.4(4.0)$ & $7.3(3.2)$ & $-41.4 \%$ & .02 & $0.0(0.0$ to 0.0$)$ & $-2.1(-5.3$ to 1.2$)$ & $-0.5(-1.2$ to 0.2$)$ \\
\hline Traumatic injuries ${ }^{\mathrm{d}}$ & $37.3(5.3)$ & $27.3(5.3)$ & $-26.8 \%$ & .004 & $-0.1(-0.2$ to 0.0$)$ & $-4.8(-12.1$ to 2.4$)$ & $-1.4(-2.6$ to -0.1$)$ \\
\hline Chronic disease exacerbations & $49.3(5.1)$ & $22.1(8.1)$ & $-55.1 \%$ & $<.001$ & $0.0(-0.1$ to 0.1$)$ & $-17.9(-24.8$ to -11.1$)$ & $-2.6(-4.6$ to -0.6$)$ \\
\hline Heart failure & $25.1(5.1)$ & $11.7(5.8)$ & $-53.4 \%$ & $<.001$ & $0.0(-0.1$ to 0.0$)$ & $-7.0(-10.9$ to -3.2$)$ & $-1.6(-3.0$ to -0.2$)$ \\
\hline COPD/asthma & $9.0(2.4)$ & $3.4(1.6)$ & $-61.9 \%$ & $<.001$ & $0.0(0.0$ to 0.1$)$ & $-2.8(-4.6$ to -1.0$)$ & $-0.7(-0.9$ to -0.5$)$ \\
\hline Diabetes & $15.1(3.0)$ & $7.0(2.0)$ & $-53.8 \%$ & $<.001$ & $0.0(0.0$ to 0.1$)$ & $-8.1(-11.4$ to -4.8$)$ & $-0.4(-1.0$ to 0.3$)$ \\
\hline Obstetric hospitalizations & $118.7(9.5)$ & $103.4(6.8)$ & $-12.9 \%$ & .005 & $0.0(-0.2$ to 0.1$)$ & $-3.0(-15.1$ to 9.0$)$ & $-2.7(-3.5$ to -1.8$)$ \\
\hline
\end{tabular}

COPD, chronic obstructive pulmonary disease

${ }^{a}$ Cardiovascular hospitalizations include acute myocardial infarction, cardiac arrest and ventricular fibrillation, cardiac dysrhythmias, arterial dissections, endocarditis and endocardial disease, myocarditis and cardiomyopathy, and pericarditis and pericardial disease

${ }^{b}$ Intra-abdominal emergencies include appendicitis and other appendiceal conditions, gastrointestinal and biliary perforation, intestinal obstruction and ileus, peritonitis, and intra-abdominal abscess

${ }^{c}$ Acute cerebrovascular events include acute hemorrhagic cerebrovascular disease, cerebral infarction, and transient cerebral ischemia

${ }^{d}$ Traumatic injuries include initial encounters for fractures, dislocations, traumatic brain and spinal cord injury, open wounds, crush injuries, and burns

Further population-based studies are needed to determine the impact of the COVID-19 pandemic on long-term outcomes of patients delaying care for acute and chronic conditions.

Timothy S. Anderson, MD, MAS ${ }^{1,2}$

Jennifer P. Stevens, $M D, M^{2,3,4}$

Adlin Pinheiro, $M A^{1}$

Stephanie Li, $\mathrm{MS}^{4}$

Shoshana J. Herzig, MD, MPH ${ }^{1,2,4}$

${ }^{1}$ Division of General Medicine, Beth Israel Deaconess Medical Center,

Boston, MA, USA

${ }^{2}$ Harvard Medical School,

Boston, MA, USA

${ }^{3}$ Division of Pulmonary, Critical Care and Sleep

Medicine, Beth Israel Deaconess Medical Center,

Boston, MA, USA

${ }^{4}$ Center for Healthcare Delivery Science, Beth Israel Deaconess Medical Center,

Boston, MA, USA

Corresponding Author: Timothy S. Anderson, MD, MAS; Division of General Medicine, Beth Israel Deaconess Medical Center, Boston, MA, USA (e-mail: tsander1@bidmc.harvard.edu).

Author Contributions Dr. Anderson had full access to all the data in the study and takes responsibility for the integrity of the data and the accuracy of the data analysis.

Study concept and design: Anderson, Herzig.
Acquisition, analysis, and interpretation of data: All authors. Drafting of the manuscript: Anderson.

Critical revision of the manuscript for important intellectual content: All authors.

Statistical analysis: Pinheiro, Anderson.

Obtained funding: N/A.

Administrative, technical, or material support: Stevens, Herzig.

Study supervision: Stevens, Herzig.

\section{Compliance with Ethical Standards:}

This research was classified as exempt by the Beth Israel Deaconess Medical Center institutional review board.

Conflict of Interest: The authors declare that they do not have a conflict of interest.

Disclaimer: The views expressed herein are those of the authors and do not necessarily represent the views of Beth Israel Deaconess Medical Center.

\section{REFERENCES}

1. Hamel L, Kearney A, Kirzinger A, et al. KFF Health Tracking Poll May 2020: Impact of Coronavirus on Personal Health, Economic and Food Security, and Medicaid. https://www.kff.org/report-section/kff-healthtracking-poll-may-2020-health-and-economic-impacts/. Published May 27, 2020. Accessed May 28, 2020.

2. Strata Decision Science. National Patient and Procedure Volume Tracker. https://www.stratadecision.com/wp-content/uploads/2020/05/National-Patient-and-Procedure-Volume-Tracker-and-Report_May2020.pdf. Published May 2020. Accessed May 18, 2020.

3. Bhatt AS, Moscone A, McElrath EE, et al. Declines in Hospitalizations for Acute Cardiovascular Conditions During the COVID-19 Pandemic: A Multicenter Tertiary Care Experience. J Am Coll Cardiol. 2020;22. https:// doi.org/10.1016/j.jacc.2020.05.038 
4. Solomon MD, McNulty EJ, Rana JS, et al. The Covid-19 Pandemic and the Incidence of Acute Myocardial Infarction [published online ahead of print, 2020 May 19]. N Engl J Med. 2020; doi:https://doi.org/10.1056/ NEJMc2015630.

5. Healthcare Cost and Utilization Project. Clinical Classifications Software Refined (CCSR) for ICD-10-CM Diagnoses. www.hcup-us.ahrq.gov/ toolssoftware/ccsr/ccs_refined.jsp. Published February 2020. Accessed May $10^{\text {th }}, 2020$.
6. Baum A, Schwartz MD. Admissions to Veterans Affairs Hospitals for Emergency Conditions During the COVID-19 Pandemic. JAMA. 2020; doi:https://doi.org/10.1001/jama.2020.9972

Publisher's Note: Springer Nature remains neutral with regard to jurisdictional claims in published maps and institutional affiliations. 